\title{
Histoire locale et mémoire ouvrière. À propos d'un livre récent
}

L'arsenal de Rennes de 1793 à nos jours. De l'histoire industrielle à la naissance d'un quartier, Association « Mémoire Arsenal-Courrouze » avec Christine Barbedet, Saint-Jacques-de-la-Lande, Les éditions de Juillet, 2012, 258 p.

Local history and working-class memory. Remarks on a recent book

Jérôme Cucarull

\section{Q OpenEdition}

Journals

Édition électronique

URL : http://journals.openedition.org/abpo/2741

DOI : 10.4000/abpo. 2741

ISBN : 978-2-7535-3407-0

ISSN : 2108-6443

Éditeur

Presses universitaires de Rennes

Édition imprimée

Date de publication : 27 mars 2014

Pagination : 187-191

ISBN : 978-2-7535-3405-6

ISSN : 0399-0826

Référence électronique

Jérôme Cucarull, « Histoire locale et mémoire ouvrière. À propos d'un livre récent », Annales de Bretagne et des Pays de l'Ouest [En ligne], 121-1 | 2014, mis en ligne le 27 mars 2016, consulté le 21 août 2020. URL : http://journals.openedition.org/abpo/2741 ; DOI : https://doi.org/10.4000/abpo.2741 


\title{
Histoire locale et mémoire ouvrière À propos d'un livre récent
}

\author{
L'arsenal de Rennes de 1793 à nos jours. \\ De l'histoire industrielle à la naissance d'un quartier, \\ Association "Mémoire Arsenal-Courrouze " \\ avec Christine Barbedet, Les éditions de Juillet, \\ Saint-Jacques-de-la-Lande, 2012, 258 p.
}

\author{
Jérôme CUCARULL \\ Chercheur associé au CERHIO (UMR CNRS 6258)
}

\begin{abstract}
Après avoir travaillé en tant que consultant dans le cadre de la réflexion sur l'aménagement de l'espace occupé par l'ancien Arsenal de Rennes, sur le site duquel émerge progressivement un nouveau quartier ("la Courrouze "), Jérôme Cucarull, chercheur associé au CERHIO, a été amené à en restituer l'histoire. Il revient sur le livre destiné au grand public réalisé par une association d'anciens ouvriers aidés d'une journaliste, Christine Barbedet, et d'un photographe, Richard Volante. Il propose ce faisant une réflexion sur la frontière entre histoire et mémoire, en particulier quand se profile, en toile de fond, la question des implications politiques des choix opérés pour justifier l'identité d'un projet de développement urbain.
\end{abstract}

Voilà une monographie qui comble un manque car l'histoire des arsenaux terrestres français est mal connue, surtout comparée à celle des arsenaux de la marine, dont le prestige est incomparable ${ }^{1}$. Parmi les établissements ayant fait l'objet d'une monographie, l'arsenal de Rennes est probablement le plus mal documenté. Non seulement les sources sont

1. La thèse de Patrice Mortal a marqué une première avancée dans ce domaine mais ce travail reste isolé. MoRTAL, Patrick, Les armuriers de l'État du Grand Siècle à la globalisation 1665-1989, Presses Universitaires du Septentrion, Villeneuve-d'Asq, 2007. Il existe néanmoins un certain nombre de monographies d'établissement que l'on trouvera dans la bibliographie de l'ouvrage. 
éparses, mais les archives de l'établissement ont été dans leur grande majorité détruites peu avant sa fermeture et le fonds, qui a été classé par le Centre des archives de l'armement et du personnel de Châtellerault en 2008 (côte 3H7), est essentiellement composé de plans (1485 recensés). Ceci explique que jusqu'à la Première Guerre mondiale on possède peu de détails sur cette histoire, et cette période ne couvre d'ailleurs que 20 pages dans l'ouvrage.

\section{Une histoire redécouverte}

La nécessité de former, dans l'ouest de la France, un établissement d'Artillerie date du XVIII ${ }^{\mathrm{e}}$ siècle, notamment lorsque l'Angleterre fait peser la menace d'une invasion sur la Bretagne en 1778. Mais les projets n'aboutissent qu'en 1793, quand la Convention décide de créer à Rennes un " arsenal de construction et de dépôt destiné à l'approvisionnement des places et batteries de côte ". Rennes devient ainsi une plate-forme logistique pour les militaires. Cependant, du fait du manque de fonds disponibles, la première pierre d'un véritable arsenal n'est posée qu'en 1844.

$\mathrm{Au} \mathrm{XIX}^{\mathrm{e}}$ siècle, le contexte est globalement favorable au développement de la production industrielle d'armement. Après la guerre de 1870-1871, une modernisation de l'armement intervient et la rationalisation des productions est opérée. En 1902 est créé un bureau central chargé de la comptabilité générale et en 1906, un atelier central d'outillage réunit les ateliers jusqu'alors affectés chacun à une fabrication particulière.

Les relations entre la ville et l'arsenal deviennent rapidement un sujet de plus en plus préoccupant. Rennes est en plein développement et l'arsenal qui était relativement isolé a été progressivement rattrapé par elle. Il faut alors organiser la cohabitation, et des conférences sont réunies pour résoudre les difficultés qui surgissent. Cette urbanisation oblige finalement l'établissement à se développer sur deux sites. En 1856, il acquiert un terrain hors de la ville, au lieu dit la Courrouze, pour construire des magasins à poudre et y transporter les munitions. Dans les décennies 1870 et 1880, des acquisitions permettent d'y concentrer les activités dangereuses liées au chargement et aux manipulations des poudres et des artifices.

L'établissement est à son apogée durant la Première Guerre mondiale. À partir de 1916, on assiste à une extension et un renforcement sans précédent des installations. L'arsenal de Rennes joue alors un rôle essentiel pour la fourniture des munitions au front. L'effectif qui, à la veille de la guerre était de 1300 ouvriers atteint plus de 18000 en 1917. Pour renforcer la main d'œuvre locale, 2000 travailleurs coloniaux et étrangers sont recrutés. Ils sont hébergés dans les baraquements, les camps dits de Verdun et de la Marne, aménagés près de la Courrouze.

Après la fin des hostilités, l'établissement se trouve en sur-capacité. Un certain nombre de terrains non utilisés sont donc revendus ou mis en location. Le camp de Verdun est occupé par des réfugiés espagnols. Puis, 
face aux besoins engendrés par le réarmement tardif de la France, en 1938 l'arsenal s'étend à nouveau, sur plus de 24 hectares. De gros investissements sont faits mais les constructions sont inachevées au moment de la déclaration de guerre.

Du 18 juin 1940 au 4 août 1944, l'Atelier de Construction est occupé par l'armée allemande. L'établissement est utilisé par l'occupant pour y abriter ses services. Les machines les plus modernes et des stocks importants de matières premières sont expédiés en Allemagne. Grâce à des archives inédites collectées par les auteurs, le livre montre clairement comment l'arsenal a passé cette période, en multipliant les actes de résistance à l'occupant.

La période qui va de l'après-guerre à la fermeture du site occupe la majeure partie de l'ouvrage (108 pages). Dans un premier temps, dans l'attente d'un programme de fabrication d'armement, en vue d'occuper son personnel et pour répondre aux besoins du secteur privé, l'établissement exécute des commandes civiles. C'est Charles Tillon, ancien ajusteur de l'arsenal devenu ministre de l'Armement en novembre 1945, qui lance ce programme. On y fabrique des tours d'horloger, des batteuses, des semoirs, etc. Les productions militaires ne représentent plus alors que $14 \%$ de l'activité.

Puis peu à peu l'arsenal retrouve son activité première. En 1947, est installée à l'Arsenal-Ville, une nouvelle fonderie mécanisée. À la Courrouze, une douillerie mécanique pour produire des douilles en acier est construite en 1953. Les presses qui l'équipent ont été récupérées à Ebange en Moselle. Le site dispose désormais d'un équipement moderne et la cessation d'activité de la douillerie de Mulhouse fait de Rennes le seul centre de production en France. En 1958, il occupe 970 personnes, la valeur globale de la production s'élève à 5,3 milliards de francs.

Mais dans le même temps l'entreprise se restructure. Des opérations d'envergure pour la cession des terrains ont lieu dans les décennies 1950 et 1960. Certains, comme les Camp de la Marne et de Verdun sont remis aux domaines, d'autres sont cédés à la ville de Saint-Jacques-de-la-Lande. Parallèlement, des structures civiles comme le Centre d'Appareillage des Mutilés ou la Caisse régionale de Sécurité Sociale occupent provisoirement des bâtiments vides.

Au début des années 1960, des rumeurs se propagent concernant la fermeture programmée de l'Arsenal et la migration des productions vers l'industrie privée. L'Arsenal Ville est vétuste. Une refonte totale de l'établissement est donc décidée : toutes les installations sont regroupées à la Courrouze. En raison de l'extension de la ville, on abandonne le chargement des douilles en explosif, transféré à Salbris. Pour s'adapter, l'arsenal élargit ses productions. Il réalise des remorques pour l'Armée, des appareils de décontamination, ainsi que des shelters pour la maintenance des chars. L'établissement rejoint le Groupement Industriel des Armements Terrestres 
(GIAT) en 1971. Mais la production diminue progressivement. Malgré les efforts des syndicats pour le maintien de l'entreprise, qui se traduit par un conflit social long et dur, l'arsenal ferme définitivement en 2000.

\section{Un récit entre histoire et mémoire}

Même si la démarche est chronologique, l'objectif de ce livre n'est pas un travail à proprement parler historique. Il veut faire acte de mémoire alors que le site est actuellement en cours d'effacement ${ }^{2}$, comme le précise clairement la préambule de Jean-Claude Hamelin, ancien ouvrier de l'arsenal et président de l'association " Mémoire Arsenal-Courrouze " qui s'est constituée spécialement pour réaliser ce livre. C'est ainsi qu'on va y trouver concentrées les caractéristiques propres à ce type de démarche qui depuis deux décennies interpelle les historiens et tous ceux qui s'interrogent sur le sens à donner au passé et sur la manière dont il est reconstruit ${ }^{3}$.

Il y a d'abord une spécificité dans les sources utilisées. Les souvenirs constituent la base de l'information fournie à partir de la décennie 1950 . Il y a alors une connexion étroite entre les souvenirs, recoupés ou non avec d'autres témoignages, et les données fournies par des documents d'archives, dont une partie est privée et une autre invérifiable car elles ont été reproduites par un cadre de l'entreprise avant la destruction des archives de l'arsenal. Dans le même ordre d'idée, seule l'iconographie provenant de fonds publics comportent des indications de sources, et beaucoup d'images ne sont pas datées, même de manière approximative.

Cet objectif mémoriel a des incidences non négligeables sur la manière dont a été restituée l'histoire du site. Ainsi, certaines informations concernant la Seconde Guerre mondiale trouvées dans des archives inédites n'ont pas été publiées. L'engagement des auteurs est décelable dans le choix de la présentation, avec un balancement permanent entre histoire générale et histoire syndicale. Ainsi, pour la période allant des années 1945 à 1987 chacun des 4 chapitres comporte une partie appelée " syndicat et vie sociale " qui représente la moitié du texte. Ensuite, pour la période postérieure à 1987, les choses sont mêlées comme si, avec la montée des inquiétudes et la fin programmée de l'établissement il n'y avait plus de barrière entre sa vie propre et l'action syndicale. On retrouve ici l'histoire "totémique " chère à Pierre Nora, caractérisée par une représentation émotionnelle du passé par un groupe qui, par l'intermédiaire de cette remémoration subjective, tente de se faire une place dans un espace urbain en recomposition.

2. Un nouveau quartier, qui comprendra à terme 10000 habitants, sort progressivement de terre.

3. FrANK, Robert, "La mémoire et l'histoire ", Cahier de l'IHTP n 21 : La bouche de la Vérité? La recherche historique et les sources orales, nov. 1992 (consultable en ligne : [http://www.ihtp.cnrs.fr/spip.php\%3Farticle233\&lang=fr.html]); RICEUR, Paul, La mémoire, l'histoire, l'oubli, Paris, Le seuil, 2000; Morin, Mélissa S. et NoËL, Patrick-Michel, "Les représentations du passé ", Conserveries mémorielles, consulté en ligne le 4 juillet 2013 [http://cm.revues.org/846]. 
La connaissance interpersonnelle devient un critère d'historicité comme le démontre le récit d'un " accident mortel à Tarbes " qui a touché un ancien ouvrier de Rennes (p. 186).

Le manque de contextualisation dans un cadre national rend parfois le propos abscons, d'autant plus que le découpage chronologique très serré fait la part belle aux ruptures plus qu'aux continuités. À l'opposé, lorsque les informations manquent et qu'il n'y a plus de mémoire vive, on trouve des évocations sans rapport évident avec le sujet. L'évocation de l'Affaire Dreyfus (p. 20-21) ou certains récits de la Première Guerre mondiale (p. 39-40) sont hors de propos pour l'historien, alors qu'elles sont importantes pour les auteurs. Cela montre les limites de la restitution de l'histoire par ses acteurs, aussi scrupuleuse et honnête soit-elle.

De plus, se pose en filigrane la question de la relation qu'une société entretient avec les vestiges de son passé. En effet, l'ouvrage offre un bel exemple du fait que "l'ouvrier ne fait pas patrimoine ${ }^{4}$ ". La fin de l'ouvrage tente de faire le lien entre des vestiges du site et des anecdotes dont sont porteurs les témoins, au travers de sept portraits photographiques réalisés en diverses parties du site en relation avec une histoire racontée par un ancien ouvrier. Mais cela reste anecdotique.

Enfin, deux entretiens, l'un avec l'élu de Rennes-métropole qui porte le projet et l'autre avec l'urbaniste chargé de transformer le site en un nouveau quartier, permettent de justifier un lien entre passé et avenir sans cependant apporter de regard critique sur cette évolution. Or il y aurait beaucoup à dire sur l'ambiguïté de la relation entre le travail historique et la résurrection de la mémoire de ce lieu en vue d'une valorisation ultérieure dans le projet urbain ${ }^{5}$. La préface rédigée par Edmond Hervé, figure tutélaire de la politique rennaise depuis plus de 35 ans, qui a initié ce projet urbain, concourt à la légitimation de ce travail local de mémoire dans une perspective proprement politique.

Finalement, ce travail d'écriture est une étape importante de la redécouverte et de la prise de conscience du rôle important que le site a joué dans l'histoire militaire et industrielle française, en attendant une approche scientifique plus globale.

4. Pour reprendre le titre d'un article d'Anne-Françoise GARÇON, « L'ouvrier ne fait pas patrimoine. De la difficulté en France de faire se rejoindre mémoire du travail et archéologie industrielle ", L'Archéologie industrielle. Revue du Cilac, n ${ }^{\circ} 36$, juin 2000, p. 48-59.

5. Cucarull, Jérôme, "Sous la Courrouze la mémoire de l'arsenal de Rennes ", Place Publique, $\mathrm{n}^{\circ}$ 8, novembre-décembre 2010, p. 69-75. 